\title{
Raman Spectroscopy of Multiphonon Emission Process in Ni-Doped PbTe
}

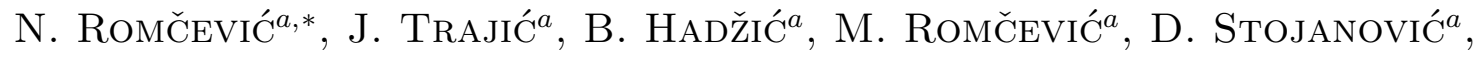 \\ Z. Lazarevićc ${ }^{a}$, T.A. Kuznetsova ${ }^{b}$, D.R. Khokhlov ${ }^{b}$, R. Rudolf ${ }^{c, d}$ And I. AnžEL ${ }^{c}$ \\ ${ }^{a}$ Institute of Physics, Pregrevica 118, 11080 Belgrade, Serbia \\ ${ }^{b}$ Moscow State University, 119899 Moscow, Russia \\ ${ }^{c}$ Faculty of Mechanical Engineering, University of Maribor, 2000 Maribor, Slovenia \\ dZlatarna Celje d.d., 3000 Celje, Slovenia
}

\begin{abstract}
In this paper, we present room temperature unpolarized Raman scattering spectra of $\mathrm{Ni}$ doped $\mathrm{PbTe}$ single crystal sample. Crystal of $\mathrm{PbTe}(\mathrm{Ni})$ was grown by the Bridgman method. The Ni concentration in the sample used here was $1 \times 10^{19}$ at. $/ \mathrm{cm}^{3}$. Well resolved peaks appear at about $126,143,181,362$ and $724 \mathrm{~cm}^{-1}$. The modes at 126 and $143 \mathrm{~cm}^{-1}$, which are also observed in other telluride compounds, originate from vibrations in $\mathrm{TeO}_{2}$. We assume that the mode at about $181 \mathrm{~cm}^{-1}$ is connected to excitations of a local phonon mode in the vicinity of an impurity atom (donor $\mathrm{Ni}^{3+}$ state). Modes at about $362 \mathrm{~cm}^{-1}$ and $724 \mathrm{~cm}^{-1}$ are the second and fourth harmonic of a local phonon mode, registered here due to multiphonon emission.
\end{abstract}

PACS numbers: 71.28.+d, 63.20.-e, 78.30.-j

\section{Introduction}

The doping of $\mathrm{A}^{\mathrm{IV}} \mathrm{B}^{\mathrm{VI}}$ semiconductor compounds with transition metal impurities has significant scientific and practical interest due to the new materials preparing possibilities. Lead telluride (PbTe) belongs to the $\mathrm{A}^{\mathrm{IV}} \mathrm{B}^{\mathrm{VI}}$ IR-sensitive narrow-gap semiconductors group, which acquires new properties as a consequence of doping $[1,2]$. Transition metals behave either as donors (Cr, Co, Ni) [3] or neutrals (Mn) [4]. For example, when PbTe is doped with chromium, free carrier concentration $n$ increases to $1.3 \times 10^{19} \mathrm{~cm}^{-3}$ and remains unchanged with further increase of the Cr concentration, up to the chromium solubility limit.

In the case of many IV-VI diluted magnetic semiconductors (DMS) deviation from stoichiometry (metal vacancies) results in the carrier density sufficiently high to produce strong ferromagnetic interactions between the localized spins. The carrier concentration can be controlled in a wide range by thermal annealing or doping.

In this paper we present Raman scattered spectra of $\mathrm{Ni}$ doped PbTe single crystal sample. According to our best knowledge this is first Raman scattering measurements of $\mathrm{PbTe}(\mathrm{Ni})$.

\section{Samples and experiment}

Single crystal of $\mathrm{PbTe}(\mathrm{Ni})$ was grown by the Bridgman method. The impurity concentration in the starting mixture was $3.3 \times 10^{20}$ at. $/ \mathrm{cm}^{3}$. The $\mathrm{Ni}$ concentration

* corresponding author; e-mail: romcevi@phy.bg.ac.yu in the crystal used here was $1 \times 10^{19}$ at. $/ \mathrm{cm}^{3}$, determined by chemical analysis [5]. The structural parameters were obtained using the X-ray diffraction (XRD) powder technique. All the registered reflections corresponded to PbTe crystals and gave the parameter of the cubic unit cell of $a=0.6455(3) \mathrm{nm}$, which is in good agreement with values cited in the literature [6]. The dislocation density was $(5-7) \times 10^{-4} \mathrm{~cm}^{-2}$, registered by selective etching [7]. The Hall effect and conductivity measurements showed that the crystal exhibits $n$-type conductivity with room-temperature electron concentration of $6 \times 10^{16} \mathrm{~cm}^{-3}$. The Raman spectra were excited by several lines of argon-laser line and measured using a Jobin Yvon model T64000 monochromator, with CCD detector.

\section{Results and discussion}

Unpolarized Raman spectra (Fig. 1) of $\mathrm{PbTe}(\mathrm{Ni}$ ) are shown in the spectral range between 30 and $1000 \mathrm{~cm}^{-1}$, at room temperature. Well resolved peaks appear at about 126, 143, 181, 362 and $724 \mathrm{~cm}^{-1}$. The modes at 126 and $143 \mathrm{~cm}^{-1}$, which are also observed in other telluride compounds, originate from vibrations in $\mathrm{TeO}_{2}$, which is always formed on the sample surface.

We assume that the mode at about $181 \mathrm{~cm}^{-1}$ is connected to excitations of a local phonon mode in the vicinity of an impurity atom (donor $\mathrm{Ni}^{3+}$ state). Modes at about $362 \mathrm{~cm}^{-1}$ and $724 \mathrm{~cm}^{-1}$ are the second and fourth harmonic of a local phonon mode, which registered here is in consequence of multiphonon emission (MPE). Namely, in the $\mathrm{PbTe}(\mathrm{Ni})$, the equilibrium position of the ground donor level before the electron capture is in the upper 


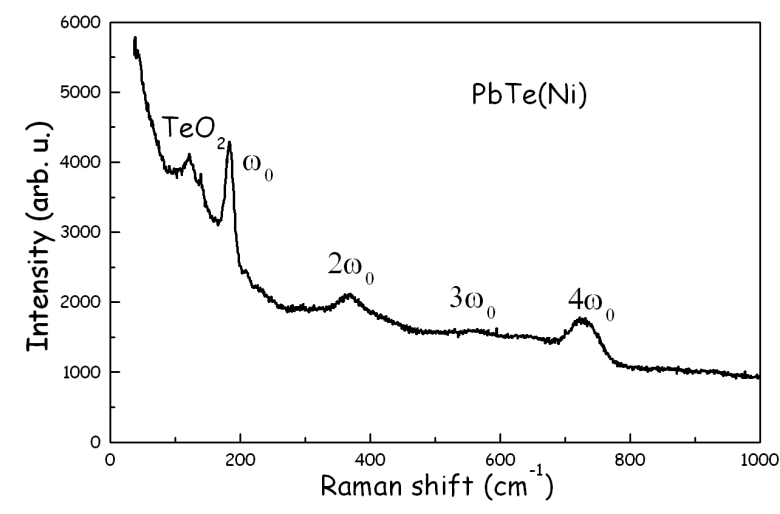

Fig. 1. Unpolarized Raman spectra of $\mathrm{PbTe}(\mathrm{Ni})$ at room temperature.

half of the gap $\left(E_{\mathrm{t}}\right)$ (Fig. 2). After the capture of the electron, the lattice near the defect relaxes in such a way lower the equilibrium position of the level in the energy gap. Immediately after the capture of the electron the lattice is displaced far from the new equilibrium position and there will be a violent lattice vibration at the defect. The vibration will rapidly damp down to the amplitude of the thermal vibrations $\left(\omega_{0}\right)$ after a small number of vibrational periods. During the damping, the localized energy propagates away from the defects as phonons. This justifies calling this process nonradiative capture by MPE [8].

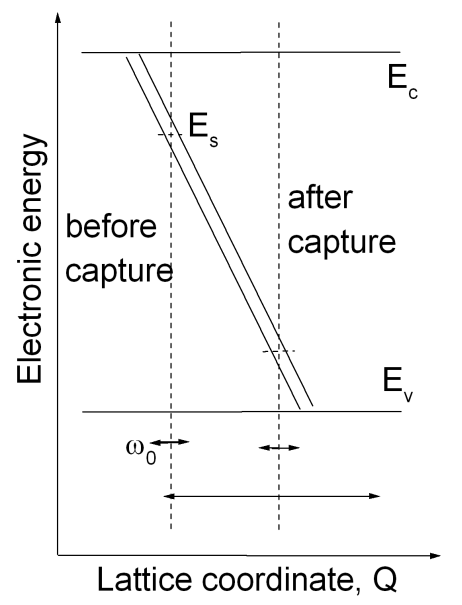

Fig. 2. Diagram illustrates how capture of an electron takes place. The smaller arrows represent the amplitude of thermal vibrations $\left(\omega_{0}\right)$. The large arrow represents the amplitude of the lattice vibrations.
The registered MPE, and effects connected with it, are of fundamental importance. Namely, it gives the direct proof that electron capture on an impurity level results in a displacement of the impurity center from the equilibrium position. As a consequence barriers are formed in configuration space between all the states of the systems with a different number of electrons.

\section{Conclusion}

In this paper, we present room temperature unpolarized Raman scattering spectra of $\mathrm{Ni}$ doped $\mathrm{PbTe}$ single crystal sample. We assume that the mode at about $181 \mathrm{~cm}^{-1}$ is connected to excitations of a local phonon mode in the vicinity of an impurity atom (donor $\mathrm{Ni}^{3+}$ state). Modes at about $362 \mathrm{~cm}^{-1}$ and $724 \mathrm{~cm}^{-1}$ are the second and fourth harmonic of a local phonon mode, registered here as according to MPE.

\section{Acknowledgments}

This work is financially supported by Serbian Ministry of Science under project 141028B.

\section{References}

[1] D.R. Khokhlov, B.A. Volkov, in: Proc. 23rd Int. Conf. on the Phys. of Semiconductors, Berlin 1996, Vol. 4, Ed. M. Schefler, World Sci., Singapore 1996, p. 2941.

[2] B.A. Volkov, O.M. Ruchaiskii, Pis'ma Zh. Eksp. Teor. Fiz. 62, 205 (1995) [JETP Lett. 62, 217 (1995)].

[3] B.A. Volkov, L.I. Ryabova, D.R. Khoklov, Physics-Uspekhi 45, 819 (2002).

[4] J. Trajić, M. Romčević, N. Romčević, A. Golubović, S. Durić, V.N. Nikiforov, J. Alloys Comp. 365, 89 (2004).

[5] V.P. Zlomanov, T.A. Kuznetsova, S.G. Dorofeev, V.D. Volodin, O.I. Tananaeva, Crystallogr. Rep. 47 (Suppl. 1), 128 (2002).

[6] JCPDS card number 38-1435.

[7] O.N. Krylyuk, A.M. Gas'kov, V.P. Zlomanov, Vestn. Mosk. Univ., Ser. 2: Khim. 6, 571 (1986).

[8] C.H. Henry, D.V. Lang, Phys. Rev. B 15, 989 (1977). 